\title{
Length difference effect on dynamic behaviors of double-walled carbon nanotubes
}

\author{
KUn $\mathrm{CAI}^{1}$, YAN Li ${ }^{1}$, HANG Yin ${ }^{1}$ AND QING HuA QIN ${ }^{2, a}$ \\ ${ }^{1}$ College of Water Resources and Architectural Engineering, Northwest A\&F University, Yangling 712100, P.R. China \\ 2 Research School of Engineering, the Australian National University, Canberra ACT, 2601, Australia
}

Received 13 March 2014, Accepted 2 August 2014

\begin{abstract}
The effect of length difference on oscillation and rotation of the inner tube in a double-walled carbon nanotube (DWNT) is investigated. In the analysis, the inner tubes of the DWNTs are assumed to be of the same length but the length of the outer tubes may be different from that of inner tubes. Further, the outer tube is entirely fixed after adequate relaxation at $300 \mathrm{~K}$ and the inner tube is unconstrained and can move freely in the outer tube. In the simulations, each bi-tube system initially has a symmetric crosssection, and the inner tube oscillates while rotating along its own axis. The dynamic behavior studied includes oscillation and rotation of the inner tubes. The oscillation decreases along with an increase in the length difference. A greater length difference between the two tubes in a bi-tube system leads to a smaller amplitude of oscillation of mass center of the inner tube (MCIT) and a higher self-rotating speed. Moreover, the inner tube in a bi-tube with different chirality of the two tubes may have different self-rotational directions.
\end{abstract}

Key words: DWNTs / length-difference effect / oscillation / rotation

\section{Introduction}

Since their discovery in 1991 [1], carbon nanotubes (CNTs) have attracted extensive attention in a variety of fields of nano-engineering [2-6] due to their superior physical properties, e.g., high strength/stiffness-todensity ratio, ultra-low friction among multi-walled carbon nanotubes (MWNTs) [7]. For example, Cumings and Zettl [7] experimentally investigated the telescopic extension of MWNTs and found ultralow inter-layer friction. In engineering application, the oscillatory [8-14] and vibrational [15-19] behaviour of CNTs has recently been reported. Zheng and Jiang [8] proposed that MWNTs could function as a gigahertz oscillator in nanoelectromechanical systems (NEMS). They also found that when the oscillation of the inner tube is activated by van der Waals force of the outer shell, the amplitude gradually decreases and the frequency increases because of the frictional force between the inner and outer tubes. Since then, effects of different parameters of MWNTs on oscillatory behaviors have been investigated [10-13]. For example, Rivera et al. $[13,14]$ analyzed the oscillatory behavior of incommensurate and commensurate double-walled carbon nanotubes (DWNTs) at different temperatures. Recently,

${ }^{a}$ Corresponding author: qinghua.qin@anu.edu.au some effective approaches have been suggested to control the behavior of oscillators [20-22]. Cox et al. [20] proposed $\mathrm{C}_{60}$-carbon nanotube oscillators that can generate frequencies as high as tens of gigahertz owing to the oscillatory motion of the $\mathrm{C}_{60}$ molecule inside the single-walled carbon nanotube. To create an oscillator with super-low damping, Neild et al. [22] exerted periodic forces on the core tube of DWNTs.

Besides axial gigahertz oscillator, DWNTs and MWNTs are widely used as nano resonator with radial vibrational function. Investigations on this topic have been conducted both theoretically [15-17] and experimentally $[18,19]$. For example, Li and Kardomateas [17] proposed a nonlocal shell model of MWNTs embedded in an elastic media to study vibrational behavior at small length scale. The results show that natural frequencies can be influenced significantly by their geometric sizes with inverse relationship. Liu et al. [19] studied radial breathing mode oscillation of DWNTs with different chirality through TEM and laser beam.

The above studies show that the oscillatory behavior of MWNTs depends basically on two factors: one is the friction between adjacent walls; the other is the end-effect, i.e., the attraction at the ends of outer tubes to the inner tube(s). In particular, Xia and Curtin [23] used a molecular dynamic (MD) method to calculate the pullout force 
(a) $4.24 \mathrm{~nm} / 980$ atoms

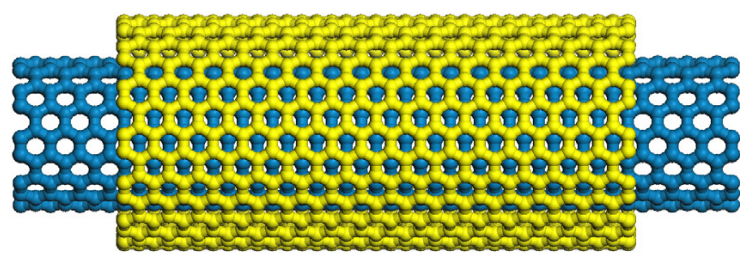

(c) $3.74 \mathrm{~nm} / 868$ atoms

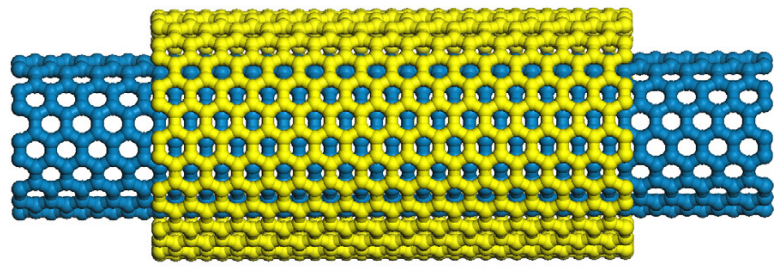

(b) $3.99 \mathrm{~nm} / 924$ atoms

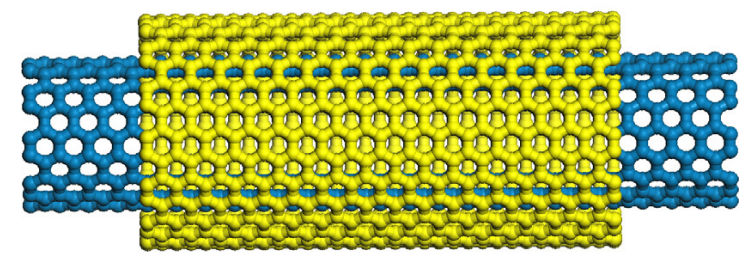

(d) $2.00 \mathrm{~nm} / 470$ atoms

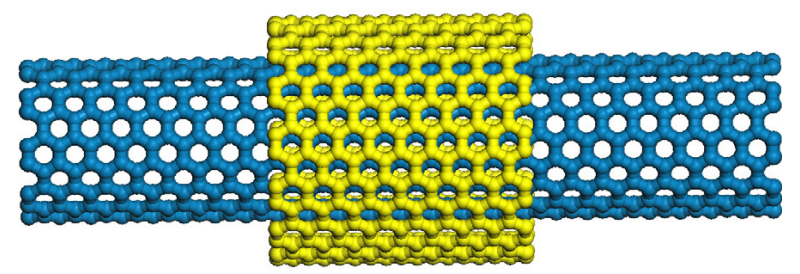

Fig. 1. Four geometries of DWNTs with chirality of $(9,9) /(14,14)$, length of $5.99 \mathrm{~nm}$ for all inner tubes and outer tubes of different lengths: (a) $4.24 \mathrm{~nm}$, (b) $3.99 \mathrm{~nm}$, (c) $3.74 \mathrm{~nm}$ and (d) $2.00 \mathrm{~nm}$.

and friction of DWNTs, demonstrating that the pullout forces of inner tube walls with fractured ends were almost three to four times greater than those of inner tube walls with capped ends. Therefore, end-effect plays an important role in the dynamic properties of CNTs. To our knowledge, however, no accurate continuum mechanics solution is available in the literature for DWNTs with different lengths between inner and outer tubes. There is also no accurate shell model for the description of the present DWCNT system. It is may be due to the difficulty in handling the different lengths of walls in DWCNT and the interaction of atoms at the ends of walls being different from that in the mid-part of walls in our recent motor trans-systems. In the present work, we will present a systematic study of the length-difference effect of DWNTs on their oscillatory and rotational behavior using MD method.

\section{Physical models and parameters}

In this section MD simulations are presented to demonstrate the length difference effect of DWNTs. In the simulations, the AIREBO potential [24] is used to evaluate the interaction between adjacent carbon atoms and the Verlet integration scheme is employed using a time-step size of $1 \mathrm{fs}$. As for oscillation and rotation frequencies in this work are at gigahertz level, 1 femtosecond is small enough to achieve satisfactory accuracy in the present simulations. Figure 1 shows four different DWNTs, $(9,9) /(14,14)$, in which the length of the inner tube is $5.99 \mathrm{~nm}$ with 882 carbon atoms and the lengths of outer tubes (i.e., $(14,14)$ ) are $4.24 \mathrm{~nm}$, $3.99 \mathrm{~nm}, 3.74 \mathrm{~nm}$ and $2.00 \mathrm{~nm}$, respectively. The numbers of atoms in the outer tubes are 980,924, 868 and 476 for the different lengths, respectively. To study the effect of chirality on the dynamic behavior of DWNTs, an example of $(13,5) /(20,7)$ DWNTs is also considered.

The classical molecular dynamics code of LAMMPS [25] is used in all the following simulations on a computer with 6 -core $(2.0 \mathrm{GHz})$ and $32 \mathrm{~GB}$ RAM. After 40 ps of relaxation of the system under a Nose-Hoover style thermostat at $300 \mathrm{~K}$, the outer tubes are entirely fixed and the entire inner tubes are unconstrained.

\section{Results and discussions}

\subsection{Oscillation behavior of inner tubes}

Figure 2 shows the oscillatory behavior of mass centers of inner tubes. As the length difference between the inner and outer tubes increases, the distance between the MCIT zigzagging and the symmetric plane of the outer tube (broken lines in Fig. 2) increases, but the degree of oscillation decreases (see Fig. 2). The major reason is that the oscillation of the potential energy (Fig. 3c) varies within a very small range. If the length of outer tube is far less than that of the inner tube, as in the bi-tubes in Figure $2 \mathrm{~d}$, the MCIT usually stays at one side of the symmetric plane of the outer tube and the oscillating amplitude of MCIT tends to be very small. It can be seen from Figure $3 c$ that the decrease in potential energy of the bi-tube system increases more quickly when the length difference between the inner and outer tubes increases. However, it is uncertain at which side of the symmetric plane of the outer tube the MCIT will stay (see Fig. 2, the MCIT is at the upper side for cases (b) and (d), and at the lower side for case (c)). These phenomena mentioned can also be found in Figure 4II, in which the $(13,5) /(20,7)$ DWNT is involved. 


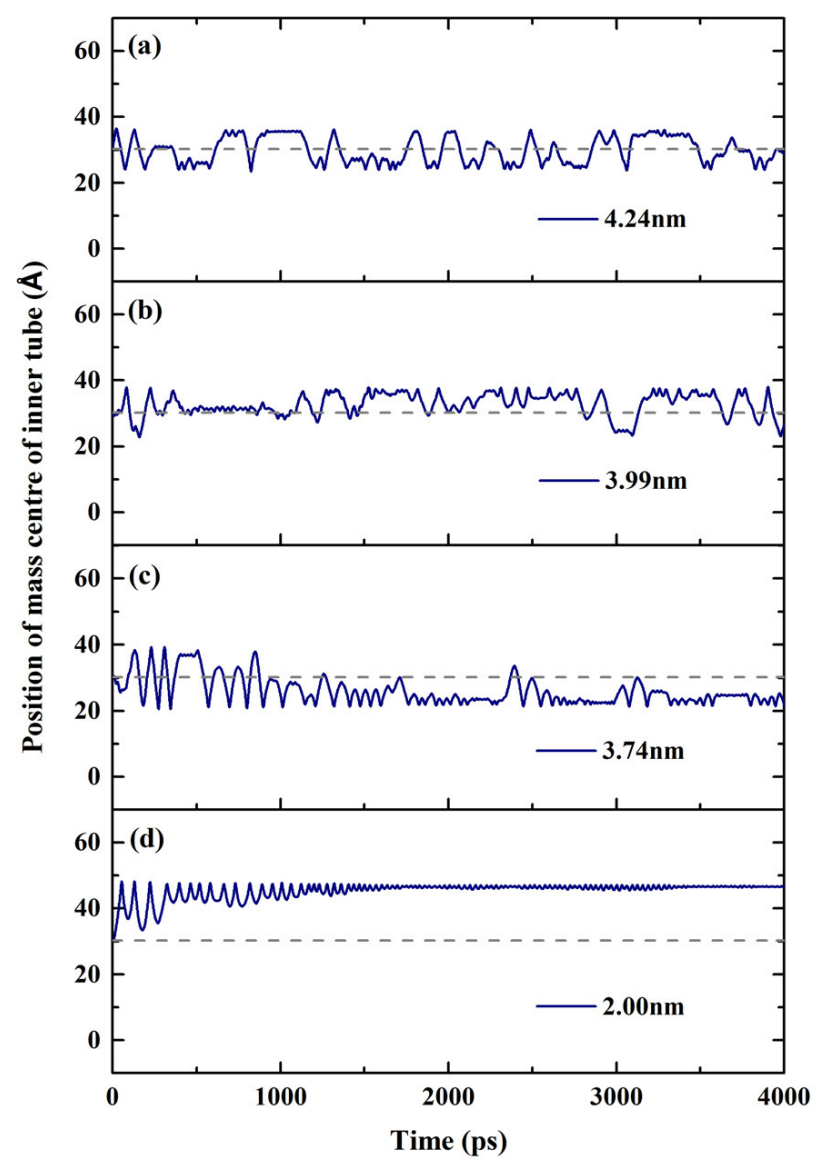

Fig. 2. Position of mass centre of inner tube (MCIT) of four DWNTs with different outer tube lengths.

\subsection{Rotational behavior of inner tube}

In addition to oscillating, the inner tube also rotates around its own axis in the length direction. After relaxation of DWNTs, inner tubes perform asymmetry configuration. Some of the atoms are close to the outer tubes and the repulsive force applied on such atoms increases sharply. Then after several steps of simulation, the rotation of inner tubes is observed. For example, the rotational frequency of the inner tube in the $2 \mathrm{~nm}$ outer tube (Fig. 3a) changes most quickly and tends to be within $[168,171] \mathrm{GHz}$ after $2530 \mathrm{ps}$. As the length of the outer tube increases, the rotational speed of the inner tube decreases, and the time needed for the inner tube to approach stable rotation increases. For instance, the stable rotating speed reaches $[120,132] \mathrm{GHz}$ after 3500 ps for the bi-tube with the $3.74 \mathrm{~nm}$ outer tube, and [75.5, 83.5] $\mathrm{GHz}$ after $6000 \mathrm{ps}$ for that with the $3.99 \mathrm{~nm}$ outer tube (see Fig. 4I). The average rotational velocity of the inner tube within the $4.24 \mathrm{~nm}$ outer tube is zero, as verified in Figure 3b. For the bi-tube with the $3.99 \mathrm{~nm}$ outer tube, the rotational speed increases monotonously, starting from 1430 ps. It can be seen from Figure 3 that the greater in decrease in potential energy, the higher is the

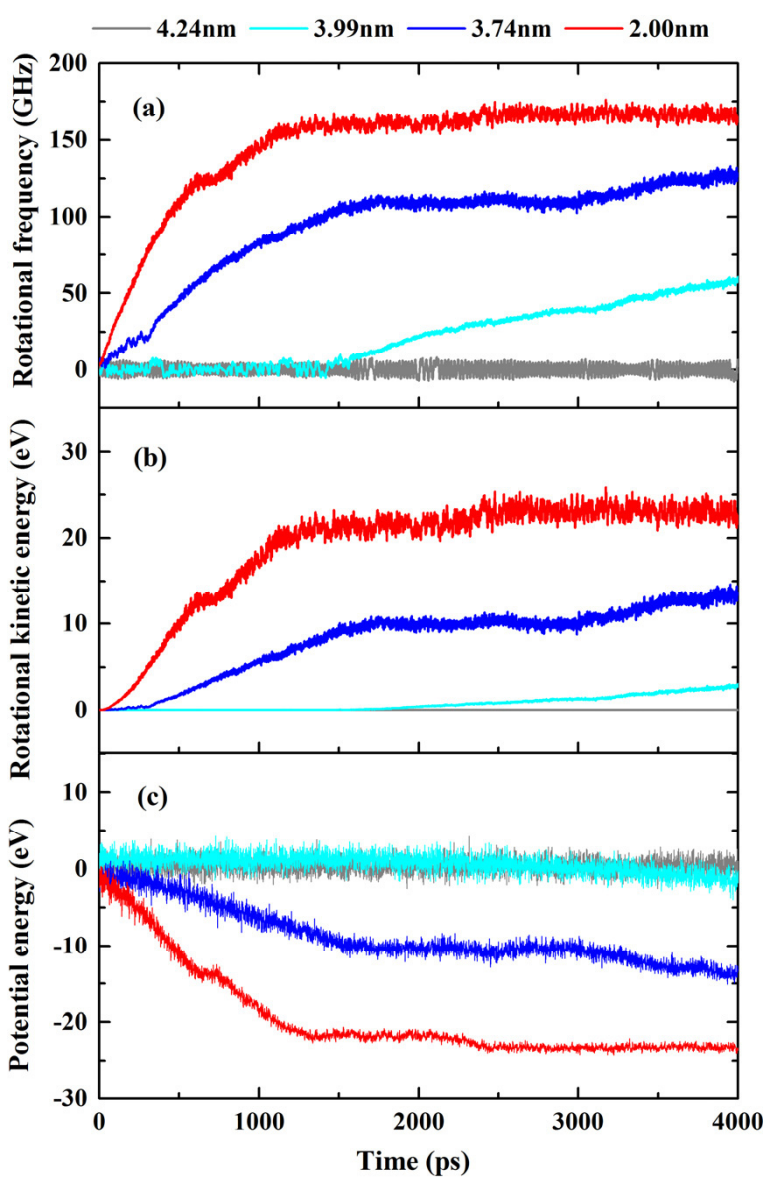

Fig. 3. Rotational frequency, energy and potential energy of four DWNTs with different outer tube lengths.

excited rotational speed of the inner tube after a shorter time.

Furthermore, as viewed from right to left of the DWNT, we found that the rotation of inner tube is counterclockwise around its own axis for the cases of Figures $1 \mathrm{~b}-1 \mathrm{~d}$. In contrast, the inner tube rotates clockwise for the case of the $(13,5) /(10,7)$ bi-tube with $2.18 \mathrm{~nm}$ outer tube and $6.00 \mathrm{~nm}$ inner tube.

\section{Conclusions}

The end-effect on the dynamic behavior of DWNTs is investigated using the classical molecular dynamics code of LAMMPS. These DWNTs consist of inner tubes of the same length and outer tubes of different lengths. The inner tubes show both rotation along their own axis and oscillation within the outer tubes when the temperature is at $300 \mathrm{~K}$. As the length difference between the two tubes in the bi-tube system increases, the amplitude of oscillation of the MCIT decreases (tending to zero in the case of Fig. 2d) and the rotational speed increases. The dynamic behaviors of rotation and oscillation depend directly on changes in the system's potential energy. 


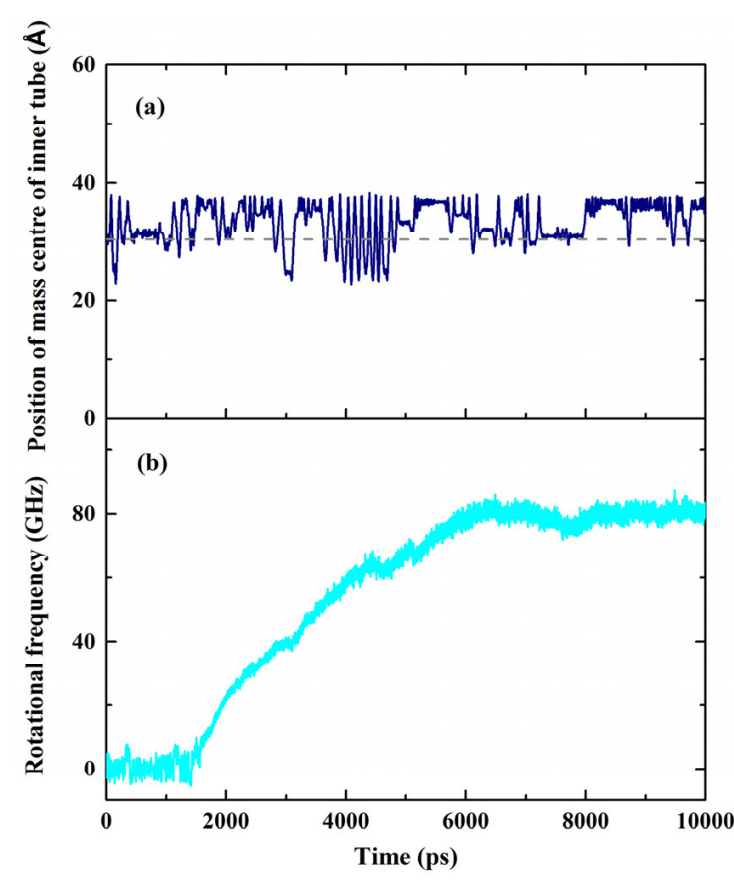

(I) $(9,9) /(14,14)$ system with $3.99 \mathrm{~nm}$
length of outer tube

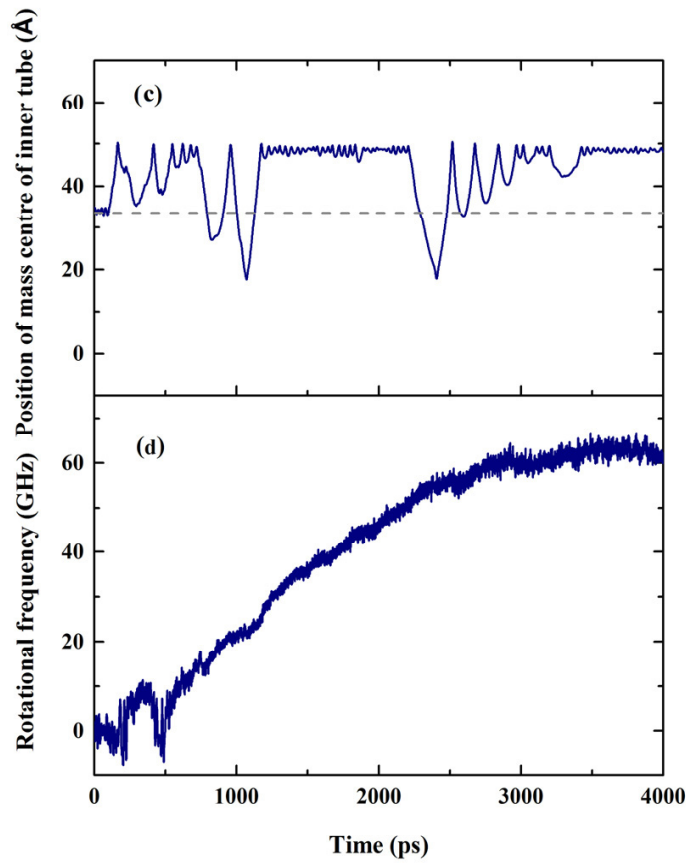

(II) $(13,5) /(20,7)$ system with $2.18 \mathrm{~nm}$ length of outer tube

Fig. 4. Dynamic behavior of the inner tube in DWNTs systems with $6.00 \mathrm{~nm}$ length of inner tube at 300 K: (I) (9,9)/(14,14) bi-tube system; (II) $(13,5) /(20,7)$ bi-tube system.

Acknowledgements. This study was partially supported by the National Natural Science Foundation of China (Grant No. 50908190), the Youth Talents Foundation of Shanxi Province (Grant No. 2011KJXX02), the Fundamental Research Funds for the Central Universities (QN2011125), and Open Research Foundation (Grant No. GZ1205) of the State Key Laboratory of Structural Analysis for Industrial Equipment, Dalian University of Technology, China.

\section{References}

[1] S. Iijima, Helical microtubules of graphitic carbon, Nature 354 (1991) 56-58

[2] Y. Nakayama, S. Akita, Nanoengineering of carbon nanotubes for nanotools, New J. Phys. 5 (2003) 128

[3] W. Qiu, Y.L. Kang, Z. Lei, Q.H. Qin, Q. Li, A new theoretical model of a carbon nanotube strain sensor, Chin. Phys. Lett. 26 (2009) 080701

[4] V. Sazonova, Y. Yaish, H. Ustunel, D. Roundy, T.A. Arias, P.L. McEuen, A tunable carbon nanotube electromechanical oscillator, Nature 431 (2004) 284-287

[5] Z. Qin, Q.H. Qin, X.Q. Feng, Mechanical property of carbon nanotubes with intramolecular junctions: Molecular dynamics simulations, Phys. Lett. A 372 (2008) 66616666

[6] W. Qiu, Y.L. Kang, Z.K. Lei, Q.H. Qin, Q. Li, Q. Wang, Experimental study of the Raman strain rosette based on the carbon nanotube strain sensor, J. Raman Spectrosc. 41 (2010) 1216-1220
[7] J. Cumings, A. Zettl, Low-friction nanoscale linear bearing realized from multiwall carbon nanotubes, Science 289 (2000) 602-604

[8] Q.S. Zheng, Q. Jiang, Multiwalled carbon nanotubes as gigahertz oscillators, Phys. Rev. Lett. 88 (2002) 045503

[9] K. Cai, H. Yin, Q.H. Qin, Y. Li, Self-excited Oscillation of Rotating Double-walled Carbon Nanotubes, Nano Lett. 14 (2014) 2558-2562

[10] W.L. Guo, Y.F. Guo, H.J. Gao, Q.S. Zheng, W.Y. Zhong, Energy dissipation in gigahertz oscillators from multiwalled carbon nanotubes, Phys. Rev. Lett. 91 (2003) 125501

[11] S.B. Legoas, V.R. Coluci, S.F. Braga, P.Z. Coura, S.O. Dantas, D.S. Galvao, Molecular-dynamics simulations of carbon nanotubes as gigahertz oscillators, Phys. Rev. Lett. 90 (2003) 055504

[12] Y. Zhao, C.C. Ma, G.H. Chen, Q. Jiang, Energy dissipation mechanisms in carbon nanotube oscillators, Phys. Rev. Lett. 91 (2003) 175504

[13] J.L. Rivera, C. McCabe, P.T. Cummings, Oscillatory behavior of double-walled nanotubes under extension: A simple nanoscale damped spring, Nano Lett. 3 (2003) 1001-1005

[14] J.L. Rivera, C. McCabe, P.T. Cummings, The oscillatory damped behaviour of incommensurate double-walled carbon nanotubes, Nanotechnology 16 (2005) 186-198

[15] J. Yoon, C.Q. Ru, A. Mioduchowski, Vibration of an embedded multiwall carbon nanotube, Compos. Sci. Technol. 63 (2003) 1533-1542

[16] C.Y. Li, T.W. Chou, Vibrational behaviors of multiwalled-carbon-nanotube-based nanomechanical resonators, Appl. Phys. Lett. 84 (2004) 121-123 
K. Cai et al.: Mechanics \& Industry 16, 110 (2015)

[17] R. Li, G.A. Kardomateas, Vibration characteristics of multiwalled carbon nanotubes embedded in elastic media by a nonlocal elastic shell model, J. Appl. Mech. Trans. ASME 74 (2007) 1087-1094

[18] D. Garcia-Sanchez, A.S. Paulo, M.J. Esplandiu, F. PerezMurano, L. Forro, A. Aguasca, A. Bachtold, Mechanical detection of carbon nanotube resonator vibrations, Phys. Rev. Lett. 99 (2007) 085501

[19] K. Liu, X. Hong, M. Wu, F. Xiao, W. Wang, X. Bai, J.W. Ager, S. Aloni, A. Zettl, E. Wang, F. Wang, Quantum-coupled radial-breathing oscillations in doublewalled carbon nanotubes, Nature Commun. 4 (2013) 1375

[20] B.J. Cox, N. Thamwattana, J.M. Hill, Mechanics of atoms and fullerenes in single-walled carbon nanotubes. II. Oscillatory behaviour, 2006, Vol. 463, p. 477, Proc. Roy. Soc. a-Math. Phys. Eng. Sci. 463 (2007) 3395-3395
[21] J.W. Kang, K.-S. Kim, H.J. Hwang, O.K. Kwon, Molecular dynamics study of effects of intertube gap on frequency-controlled carbon-nanotube oscillators, Phys. Lett. A 374 (2010) 3658-3665

[22] A. Neild, T.W. Ng, Q. Zheng, Controlled driven oscillations of double-walled carbon nanotubes, Europhys. Lett. 87 (2009)

[23] Z. Xia, W.A. Curtin, Pullout forces and friction in multiwall carbon nanotubes, Phys. Rev. B 69 (2004)

[24] S.J. Stuart, A.B. Tutein, J.A. Harrison, A reactive potential for hydrocarbons with intermolecular interactions, J. Chem. Phys. 112 (2000) 6472-6486

[25] LAMMPS Molecular Dynamics Simulator, http:// lammps.sandia.gov/, 2013 